\title{
A NOTE ON A BRUNN-MINKOWSKI INEQUALITY FOR THE GAUSSIAN MEASURE
}

\author{
PIOTR NAYAR AND TOMASZ TKOCZ \\ (Communicated by Thomas Schlumprecht)
}

Abstract. We give counterexamples related to a Gaussian Brunn-Minkowski inequality and the (B) conjecture.

\section{INTRODUCTION AND NOTATION}

Let $\gamma_{n}$ be the standard Gaussian distribution on $\mathbb{R}^{n}$, i.e. the measure with the density

$$
g_{n}(x)=\frac{1}{(2 \pi)^{n / 2}} e^{-|x|^{2} / 2},
$$

where $|\cdot|$ stands for the standard Euclidean norm. A powerful tool in convex geometry is the Brunn-Minkowski inequality for Lebesgue measure (see $[\mathrm{Sch}$ for more information). Concerning the Gaussian measure, the following question has recently been posed.

Question (R. Gardner and A. Zvavitch, GZ). Let $0<\lambda<1$ and let $A$ and $B$ be closed convex sets in $\mathbb{R}^{n}$ such that $o \in A \cap B$. Is it true that

$$
\gamma_{n}(\lambda A+(1-\lambda) B)^{1 / n} \geq \lambda \gamma_{n}(A)^{1 / n}+(1-\lambda) \gamma_{n}(B)^{1 / n} ?
$$

A counterexample is given in this note. However, we believe that this question has an affirmative answer in the case of $o$-symmetric convex sets, i.e. the sets satisfying $K=-K$.

In [CFM] it is proved that for an $o$-symmetric convex set $K$ in $\mathbb{R}^{n}$ the function

$$
\mathbb{R} \ni t \mapsto \gamma_{n}\left(e^{t} K\right)
$$

is log-concave. This was conjectured by W. Banaszczyk and was popularized by R. Latała [Lat. It turns out that the (B) conjecture cannot be extended to the class of sets which are not necessarily o-symmetric yet contain the origin, as one of the sets provided in our counterexample shows.

As for the notation, we frequently use the function

$$
T(x)=\frac{1}{\sqrt{2 \pi}} \int_{x}^{\infty} e^{-t^{2} / 2} \mathrm{~d} t .
$$

Received by the editors October 11, 2011 and, in revised form, December 17, 2011 and February $1,2012$.

2010 Mathematics Subject Classification. Primary 52A40; Secondary 60G15.

Key words and phrases. Convex body, Gauss measure, Brunn-Minkowski inequality, (B) conjecture.

The first author's research was partially supported by NCN grant 2011/01/N/ST1/01839.

The second author's research was partially supported by NCN grant 2011/01/N/ST1/05960. 


\section{Counterexamples}

Now we construct the convex sets $A, B \subset \mathbb{R}^{2}$ containing the origin such that inequality (GBM) does not hold. Later on we show that for the set $B$ the (B) conjecture is not true.

Fix $\alpha \in(0, \pi / 2)$ and $\varepsilon>0$. Take

$$
\begin{aligned}
A & =\left\{(x, y) \in \mathbb{R}^{2}|y \geq| x \mid \tan \alpha\right\}, \\
B=B_{\varepsilon} & =\left\{(x, y) \in \mathbb{R}^{2}|y \geq| x \mid \tan \alpha-\varepsilon\right\}=A-(0, \varepsilon) .
\end{aligned}
$$

Clearly, $A, B$ are convex and $0 \in A \cap B$. Moreover, from the convexity of $A$ we have $\lambda A+(1-\lambda) A=A$, and therefore

$$
\lambda A+(1-\lambda) B=\lambda A+(1-\lambda)(A-(0, \varepsilon))=A-(1-\lambda)(0, \varepsilon) .
$$

Observe that

$$
\begin{aligned}
\gamma_{2}(A) & =\frac{1}{2}-\frac{\alpha}{\pi}, \\
\gamma_{2}(B) & =2 \int_{0}^{+\infty} T(x \tan \alpha-\varepsilon) \frac{1}{\sqrt{2 \pi}} e^{-x^{2} / 2} \mathrm{~d} x, \\
\gamma_{2}(\lambda A+(1-\lambda) B) & =2 \int_{0}^{+\infty} T(x \tan \alpha-\varepsilon(1-\lambda)) \frac{1}{\sqrt{2 \pi}} e^{-x^{2} / 2} \mathrm{~d} x
\end{aligned}
$$

and that these expressions are analytic functions of $\varepsilon$. We will expand these functions in $\varepsilon$ up to the order 2 . Let

$$
a_{k}=\int_{0}^{+\infty} T^{(k)}(x \tan \alpha) \frac{1}{\sqrt{2 \pi}} e^{-x^{2} / 2} \mathrm{~d} x,
$$

for $k=0,1,2$, where $T^{(k)}$ is the $k$-th derivative of $T$ (we adopt the standard notation $\left.T^{(0)}=T\right)$. We get

$$
\begin{aligned}
\gamma_{2}(A) & =2 a_{0}, \\
\gamma_{2}(B) & =2 a_{0}-2 \varepsilon a_{1}+\varepsilon^{2} a_{2}+o\left(\varepsilon^{2}\right), \\
\gamma_{2}(\lambda A+(1-\lambda) B) & =2 a_{0}-2 \varepsilon(1-\lambda) a_{1}+\varepsilon^{2}(1-\lambda)^{2} a_{2}+o\left(\varepsilon^{2}\right) .
\end{aligned}
$$

Thus

$$
\sqrt{\gamma_{2}(B)}=\sqrt{2 a_{0}}-\frac{a_{1}}{\sqrt{2 a_{0}}} \varepsilon+\left(\frac{a_{2}}{2 \sqrt{2 a_{0}}}-\frac{a_{1}^{2}}{2\left(2 a_{0}\right)^{3 / 2}}\right) \varepsilon^{2}+o\left(\varepsilon^{2}\right) .
$$

Taking $\varepsilon(1-\lambda)$ instead of $\varepsilon$ we obtain

$$
\begin{aligned}
\sqrt{\gamma_{2}(\lambda A+(1-\lambda) B)}= & \sqrt{2 a_{0}}-\frac{a_{1}}{\sqrt{2 a_{0}}}(1-\lambda) \varepsilon \\
& +\left(\frac{a_{2}}{2 \sqrt{2 a_{0}}}-\frac{a_{1}^{2}}{2\left(2 a_{0}\right)^{3 / 2}}\right)(1-\lambda)^{2} \varepsilon^{2}+o\left(\varepsilon^{2}\right)
\end{aligned}
$$

Since

$$
\begin{gathered}
\sqrt{\gamma_{2}(\lambda A+(1-\lambda) B)}-\lambda \sqrt{\gamma_{2}(A)}-(1-\lambda) \sqrt{\gamma_{2}(B)} \\
=-\lambda(1-\lambda) \frac{1}{2\left(2 a_{0}\right)^{3 / 2}}\left(2 a_{0} a_{2}-a_{1}^{2}\right) \varepsilon^{2}+o\left(\varepsilon^{2}\right),
\end{gathered}
$$

we will have a counterexample if we find $\alpha \in(0, \pi / 2)$ such that

$$
2 a_{0} a_{2}-a_{1}^{2}>0 \text {. }
$$


Recall that $a_{0}=\frac{1}{2} \gamma_{2}(A)=\frac{1}{2}\left(\frac{1}{2}-\frac{\alpha}{\pi}\right)$. The integrals that define the $a_{k}$ 's can be calculated. Namely,

$$
\begin{aligned}
a_{1} & =\int_{0}^{\infty} T^{\prime}(x \tan \alpha) \frac{e^{-x^{2} / 2}}{\sqrt{2 \pi}} \mathrm{d} x=-\frac{1}{\sqrt{2 \pi}} \frac{1}{2} \int_{\mathbb{R}} e^{-\left(1+\tan ^{2} \alpha\right) x^{2} / 2} \frac{\mathrm{d} x}{\sqrt{2 \pi}} \\
& =-\frac{1}{\sqrt{2 \pi}} \frac{1}{2 \sqrt{1+\tan ^{2} \alpha}}, \\
a_{2} & =\int_{0}^{\infty} T^{\prime \prime}(x \tan \alpha) \frac{e^{-x^{2} / 2}}{\sqrt{2 \pi}} \mathrm{d} x=\frac{1}{\sqrt{2 \pi}} \int_{0}^{\infty}(x \tan \alpha) e^{-\left(1+\tan ^{2} \alpha\right) x^{2} / 2} \frac{\mathrm{d} x}{\sqrt{2 \pi}} \\
& =\frac{1}{2 \pi} \frac{\tan \alpha}{1+\tan ^{2} \alpha} .
\end{aligned}
$$

Therefore,

$$
\begin{aligned}
2 a_{0} a_{2}-a_{1}^{2} & =2\left(\frac{1}{2}\left(\frac{1}{2}-\frac{\alpha}{\pi}\right) \cdot \frac{1}{2 \pi} \frac{\tan \alpha}{1+\tan ^{2} \alpha}\right)-\frac{1}{2 \pi} \cdot \frac{1}{4\left(1+\tan ^{2} \alpha\right)} \\
& =\frac{1}{8 \pi} \frac{1}{1+\tan ^{2} \alpha}\left(\tan \alpha\left(2-\frac{4 \alpha}{\pi}\right)-1\right)
\end{aligned}
$$

which is positive for $\alpha$ close to $\pi / 2$.

Now we turn our attention to the $(\mathrm{B})$ conjecture. We are going to check that for the set $B=B_{\varepsilon}$ the function $\mathbb{R} \ni t \mapsto \gamma_{n}\left(e^{t} B\right)$ is not log-concave, provided that $\varepsilon$ is sufficiently small. Since

$$
e^{t} B=\left\{(x, y) \in \mathbb{R}^{2}|y \geq \tan \alpha| x \mid-\varepsilon e^{t}\right\},
$$

we get

$$
\begin{aligned}
\ln \gamma_{2}\left(e^{t} B\right) & =\ln \left(2 \int_{0}^{\infty} T\left(x \tan \alpha-e^{t} \varepsilon\right) \frac{e^{-x^{2} / 2}}{\sqrt{2 \pi}} \mathrm{d} x\right) \\
& =\ln \left(2 \int_{0}^{\infty} T(x \tan \alpha) \frac{e^{-x^{2} / 2}}{\sqrt{2 \pi}} \mathrm{d} x\right)-\varepsilon e^{t} \frac{\int_{0}^{\infty} T^{\prime}(x \tan \alpha) e^{-x^{2} / 2} \mathrm{~d} x}{\int_{0}^{\infty} T(x \tan \alpha) e^{-x^{2} / 2} \mathrm{~d} x}+o(\varepsilon) .
\end{aligned}
$$

This produces the desired counterexample for sufficiently small $\varepsilon$ as the function $t \mapsto \beta e^{t}$, where

$$
\beta=-\frac{\int_{0}^{\infty} T^{\prime}(x \tan \alpha) e^{-x^{2} / 2} \mathrm{~d} x}{\int_{0}^{\infty} T(x \tan \alpha) e^{-x^{2} / 2} \mathrm{~d} x}>0
$$

is convex.

Remark. The set $B_{\varepsilon}$ which serves as a counterexample to the (B) conjecture in the nonsymmetric case works when the parameter $\alpha=0$ as well (and $\varepsilon$ is sufficiently small). Since $B_{\varepsilon}$ is simply a halfspace in this case, it shows that the symmetry of $K$ is required for log-concavity of (1.1) even in the one-dimensional case.

\section{ACKNOWLEDGEMENTS}

The authors would like to thank Professors R. Gardner and A. Zvavitch for pointing out that the constructed set may also serve as a counterexample to the (B) conjecture in the non-symmetric case. An anonymous referee deserves thanks for the Remark. 


\section{REFERENCES}

[CFM] D. Cordero-Erausquin, M. Fradelizi, and B. Maurey, The (B) conjecture for the Gaussian measure of dilates of symmetric convex sets and related problems, J. Funct. Anal. 214 (2004), no. 2, 410-427. MR2083308 (2005g:60064)

[GZ] R. J. Gardner, A. Zvavitch, Gaussian Brunn-Minkowski-type inequalities, Trans. Amer. Math. Soc. 362 (2010), no. 10, 5333-5353. MR2657682 (2011m:52021)

[Lat] R. Latała, On some inequalities for Gaussian measures, Proceedings of the International Congress of Mathematicians, Vol. II (Beijing, 2002), 813-822, Higher Ed. Press, Beijing, 2002. MR1957087 (2004b:60055)

[Sch] R. Schneider, Convex bodies: the Brunn-Minkowski theory. Encyclopedia of Mathematics and its Applications, 44. Cambridge University Press, Cambridge, 1993. MR,1216521 (94d:52007)

Institute of Mathematics, University of Warsaw, Banacha 2, 02-097 Warszawa, POLAND

E-mail address: nayar@mimuw.edu.pl

Institute of Mathematics, University of Warsaw, Banacha 2, 02-097 Warszawa, POLAND

E-mail address: t.tkocz@mimuw.edu.pl 